\title{
Thyroid function
}

Keywords: omega 3, vitamins, thyroid, mental retardation, hypothyroidism, iodine

Abbreviations: ADD, attention deficit disorder; ADHD, attention deficit hyperactivity disorder; RDA, daily recommended allowance; MCG, micrograms; BVO, brominated vegetable oil; DHA, docosahexaenoic acid; GPC, glycerophosphocholine; PS, phosphatidylserine; GAPS, gut and psychology syndrome; PPM, part per million; EPA, ecosipentanoic acid

\section{Opinion}

Recent published research document that daily supplementation of Omega 3, Vitamin DIII and Iodine enhance brain, thyroid function, memory development, optimal hormones and IQ in both children and adults. Hypothyroidism, Goiter, Mental retardation, Cretinism (severely stunted physical and mental growth and deafness due to untreated congenital hypothyroidism), Alzheimer and Certain forms of cancer can be prevented by proper supplementation of omega 3 , vitamin DIII and Iodine. Iodine is used by your thyroid gland to help regulate metabolism and development of both your skeleton and brain, among other things. But how much iodine do you need, really? There is quite a bit of contention on this issue.

Severe iodine, vitamin DIII and Omega 3 deficiency are rampant and they are all essential nutrients for proper bodily function particularly brain function. There are two types of iodine, and that you need them both: Iodine and Iodide. The latter is the salt of iodine. One of the primary differences between them is that iodine will diffuse into the cell, whereas iodide must be transported into the cell. Different tissues in your body absorb either one or the other; hence you need both for optimal health. One is not necessarily better than the other on its own.

\section{Why is hypothyroidism more prevalent in women than men?}

There is simply no question that optimizing your iodine levels is essential for thyroid health. Hypothyroidism disproportionately affects women at a rate of about 9 to 1 in the US. The reason for this is that the female hormone estrogen inhibits the absorption of iodine.

Hypothyroidism is associated with up to $80-90$ percent free estrogen levels, compared to the normal value of 40-60 percent free estrogen. Hyperthyroidism is associated with only 20 percent free estrogen levels, and low iodine intake can lead to a hyper estrogenic state comined with low progesterone during menopause. Using bioidentical hormones with progesterone, testosterone and thyroxine as well as supplementation with Omega 3 and vitamin DIII is highly recommended for women during menopause as well as for men during andropause. Your body needs iodine for more than just your thyroid but for your whole body.

Iodine is essential for thyroid hormones that are created not just in your thyroid, but also in a woman ovaries (thyroid T2), and in the white blood cells of your bone marrow? Furthermore, iodine is not only required for proper function of your thyroid. Other tissues that absorb and use large amounts of iodine include:
Volume 2 Issue I - 2018

George Grant
World organization of Natural Medicine, CEO of Academy of
Wellness, Canada

Correspondence: George Grant, World organization of Natural Medicine, CEO of Academy of Wellness, 17 Waterhouse Way, Richmond Hill, ON L4C 9H8, Ontario, Canada, Tel + I 416562-3140,Email drgrant@rogers.com

Received: January 24, 2018 | Published: February 20, 2018

Breasts, Salivary glands, Pancreas, cerebral spinal fluid, Skin Stomach, Brain and Thymus. Thymus is the most important organ to produce $\mathrm{T}$ lymphocytes which control immune system. It a small organ in the middle of the chest that feels tender for most people with autoimmune disease like fibromyalgia, lupus and MS. Activating the thymus daily by gentle massage and taking omega 3 and iodine is highly recommended. Iodine deficiency in any of these tissues will lead to dysfunction of that tissue. Hence the following symptoms could provide clues that you are not getting enough iodine in your diet. For example, iodine deficiency in:

a) Salivary glands=inability to produce saliva, producing dry mouth

b) Skin=dry skin, and lack of sweating. Three to four weeks of iodine supplementation will typically reverse this symptom, allowing your body to sweat normally again

c) Brain=reduced alertness, and lowered IQ

d) Muscles=nodules, scar tissue, pain, fibrosis, fibromyalgia

\section{How much iodine does your body need?}

Researchers have determined that the average dietary intake of iodine for Japanese women is 13.8milligrams (mg) per day. Recommended dosage of $12.5 \mathrm{mg} /$ day, especially for pregnant women to optimize their children intelligence. Iodine supplementation at higher doses resulted in children with remarkably advanced intelligence.

Hypothyroidism, which is one of the first ailments to develop in response to iodine deficiency, is indeed particularly troublesome during pregnancy. One 1999 study found that thyroid deficiency during pregnancy can lower the child IQ by about seven points. The researchers noted that for the first 12 weeks of pregnancy, before the unborn child thyroid becomes active, the mother is the sole source of thyroid hormones. Studies suggest that these hormones play an important role in brain development. Overall, compared with other children, the offspring of thyroid-deficient mothers had impaired school performance and lower scores on tests of attention, language, and visual-motor performance. There is a synergistic effect of Iodine, Omega 3 and vitamin DIII in brain development and IQ.

But pregnant women are not the only ones who need to be concerned with the iodine content of their diet. Your thyroid alone 
needs about $6 \mathrm{mg}$ of iodine per day; the breasts of a 110-pound woman will need about $5 \mathrm{mg}$ /day (larger women or women with larger breasts need more); and other body tissues, such as your adrenals, thymus, ovaries, hypothalamus, and pituitary gland, need about $2 \mathrm{mg} / \mathrm{day}$.

\section{Here are a few more interesting facts}

i. In total, the human body can hold $1,500 \mathrm{mg}$ of iodine

ii. Your thyroid can hold a maximum of $50 \mathrm{mg}$ of iodine

iii. 20 percent of the iodine in your body is held in your skin (if your skin is depleted of iodine, you will not be able to sweat). Vitamin DIII and Omega 3 also help your skin to be healthy particularly if you suffer from eczema, psoriasis or any other skin disorder.

iv. 32 percent of your body iodine stores are in your muscles (if muscles are depleted, pain and other fibromyalgia symptoms can develop)

v. It is important to realize that the current US daily recommended allowance (RDA) for iodine are not in milligram doses but in micrograms:150micrograms ( $\mathrm{mcg}$ ) per day for adult men and women, $220 \mathrm{mcg}$ for pregnant women, $290 \mathrm{mcg}$ for lactating/ breastfeeding women

However, this RDA was set with the intention to prevent goiter only. Dr. Flechas makes a compelling argument for it being completely insufficient for overall physical health and prevention of diseases such as thyroid disease, fibromyalgia, and cancer. Iodine actually induces apoptosis, meaning it causes cancer cells to self destruct.

For example, between 1971 and 2000, the average iodine levels declined by 50 percent in the US. During that same time, cancers specifically associated with iodine deficiency such as cancer of the breast, prostate, endometrium, and ovaries increased. He also points out that the RDA completely ignores the presence of increasing amounts of goitrogens in the environment. The following halogens compete for the same receptors used in your thyroid gland and elsewhere to capture iodine, so if you are exposed to too many of these, your thyroid hormone production can be severely disrupted, resulting in a low thyroid state:

Bromide / bromine (Bromide can be found in several forms. Methyl bromide is a pesticide used mainly on strawberries, found predominantly in the California areas. Brominated vegetable oil (BVO) is added to citrus drinks to help suspend the flavoring in the liquid. Potassium bromate is a dough conditioner found in commercial bakery products.

Doses of iodine as high as 12.5 milligrams $(\mathrm{mg})$ per day, which is greater from the RDA of 150 micrograms ( $\mathrm{mcg}$ ), we need more research to determine the health effects of too much iodine. Recently published study has cast some doubts on high-dose iodine supplementation. The study, published December 28, 2011 in the American Journal of Clinical Nutrition, randomly assigned one of 12 different dosages of iodine (ranging from 0 to $2,000 \mathrm{mcg} /$ day) to healthy adults for four weeks.

When diet was factored in, those taking $400 \mathrm{mcg} /$ day were receiving a total of about $800 \mathrm{mcg}$ of iodine per day. At doses at and above $400 \mathrm{mcg}$ of supplemented iodine per day, some of the study participants developed sub clinical hypothyroidism, which appeared to be dose dependent. At $400 \mathrm{mcg} /$ day, five percent developed sub clinical hypothyroidism; at the highest dose $2,000 \mathrm{mcg} /$ day 47 percent of participants were thus affected. Sub clinical hypothyroidism refers to a reduction in thyroid hormone levels that is not sufficient to produce obvious symptoms of hypothyroidism (such as fatigue, dry skin, depression or weight gain, just to mention a few symptoms).

These findings suggest it might not be wise to get more than about $800 \mathrm{mcg}$ of iodine per day, and supplementing with as much as $10 \mathrm{mg}$ $(10,000 \mathrm{mcg})$ may potentially have some adverse health effects. More research is needed to elucidate the synergistic effect of using iodine, Omega 3 and vitamin DIII together. While iodine is clearly needed for health brain development and function, it is by no means the only factor that determines your child mental capacity. Here are my recommendations for the majority of my clients:
i. Omega 3 fatty acids [DHA + EPA]
ii. Vitamin DIII
iii. Avoiding fluoride and pesticides containing bromine.
iv. Optimizing gut flora

DHA [Docosahexaenoic acid] is an essential structural component of both your brain and retina. Approximately 60 percent of your brain is composed of fats 25 percent of which is DHA. DHA is also an essential structural ingredient of breast milk, which is believed to be a major reason why breastfed babies consistently score higher on IQ tests than formula fed babies.

Omega-3 fats containing both DHA and EPA [Ecosipentanoic acid] are considered essential because your body cannot produce it, and must get it from your daily diet. Omega 3 rich foods include fish, liver, and brain all of which are no longer consumed in great amounts by most Americans. To compensate for our inherently low omega-3 diet, a high quality animal-based omega-3 supplement is something that I recommend for virtually everyone, especially if you are pregnant.

Most of the DHA needed for brain and nervous system development is transferred from the mother to the fetus during the last trimester. The DHA content in the mother diet reflects in the amount of DHA passed on to the baby. DHA levels of premature infants are especially low since they miss much of that last trimester. Preemies are also more likely to be bottle fed, hence missing out on valuable DHA from their mother's breast milk. While you can now find infant formula that contains added DHA, I do not believe it is anywhere near comparable to the DHA found in breast milk.

Studies show that low DHA intake in infancy can lead or contribute to Attention Deficit Disorder (ADD) and Attention Deficit Hyperactivity Disorder (ADHD). Low DHA levels have also been linked to increased risk of suicide and dementia.

\section{Why animal-based omega-3?}

While there are both plant and animal sources for omega-3 fats, there are differences between them, and when it comes to protecting brain function, you need the animal-based version. There are three important omega-3 fatty acids ALA, EPA and DHA. DHA is the most important for your brain. EPA is also required by your brain, but in smaller amounts.

Plant-based omega-3 sources like flax, hemp and chia seeds are high in ALA, but low in EPA and DHA. Although ALA is an essential nutrient, the key point to remember is that the conversion of ALA to 
the far more essential EPA and DHA is typically quite inhibited by impaired delta 6 desaturase, an enzyme necessary for you to convert the ALA into the longer chain EPA and DHA. Because of this, it is important to include animal-based sources of omega- 3 fats that is considered anti inflammatory vs Omega 6 which is known to increase inflammation.

\section{Vitamin d iii, omega 3, and iodine deficiency may be a primary culprit in skyrocketing autism rates}

In more recent years, rampant vitamin DIII deficiency has been linked to a proportionate jump in autism. While the connection may not be obvious, it is important to realize that vitamin DIII receptors appears in a wide variety of brain tissue during early fetal development, and activated vitamin D receptors increase nerve growth in your brain. Researchers have also located metabolic pathways for vitamin $\mathrm{D}$ in the hippocampus and cerebellum of the brain, areas that are involved in planning, processing of information, and the formation of new memories.

The National Institutes of Mental Health recently concluded that it is vital that the mother get enough vitamin DIII, Omega 3 and iodine while pregnant in order for the baby brain to develop properly. The child must also get enough vitamin D after birth for normal brain functioning. Appropriate sun exposure would take care of these issues, as the sun is irreplaceable when it comes to the body ability to produce adequate amounts of vitamin DIII.

Vitamin D deficiency is also associated with depression. In 2006, scientists evaluated the effects of vitamin D on the mental health of 80 elderly patients and found those with the lowest levels of vitamin D were 11 times more prone to be depressed than those who received healthy doses. More recently, researchers found that intake of more than $400 \mathrm{IU}$ of vitamin D from food sources was associated with a 20 percent lower risk of depressive symptoms compared with intake of less than $100 \mathrm{IU}$. This was a significant benefit from a very small amount of vitamin DIII as $400 \mathrm{IU}$ is far too low to benefit most people.

It now appears as though most adults need about 2,000 IUs of vitamin D a day in order to get their serum levels above $40 \mathrm{ng} / \mathrm{ml}$, which is the lowest they should be. Ideally your serum levels should be between 50 to $70 \mathrm{ng} / \mathrm{ml}$, and up to $100 \mathrm{ng} / \mathrm{ml}$ to treat cancer and heart disease. However, it is important to realize that there is no magic dosage when it comes to vitamin D. It is important that your serum level, so you need to get your vitamin DIII levels tested to make sure you are staying within the optimal and therapeutic ranges as indicated below.

Fluoride Found to Harm Brain Function and Lower IQ in Children. Fluoride is known to interfere with basic functions of nerve cells in your brain, and numerous animal and human studies demonstrate the damage fluoride inflicts on your brain, including your pineal gland. Shockingly considering the fact that 70 percent of the US is still fluoridating their water supplies. There are more than 25 human studies and 100 animal studies linking fluoride to brain damage and reduced IQ in children. This includes such effects as:

a. Reduction in nicotinic acetylcholine receptors, damage to the hippocampus Formation of beta-amyloid plaques (the classic brain abnormality in Alzheimer disease)

b. Reduction in lipid content Damage to the purkinje cells
Exacerbation of lesions induced by iodine deficiency Impaired antioxidant defense systems, increased uptake of aluminum, and accumulation of fluoride in the pineal gland.

c. Fluoride can also increase manganese absorption, compounding problems since manganese in drinking water has also been linked to lower IQ in children.

One of the most recent studies into the effects of water fluoridation on intellectual performance, published in December 20105, found that about 28 percent of children in the low fluoride study area scored as bright, normal or higher intelligence compared to only 8 percent in the high-fluoride area. Further, 15 percent of children in the high-fluoride city had signs of mental retardation, compared with only 6 percent in the low-fluoride city. Most alarmingly, some of these brain-damaging effects have been observed even at low levels of exposure, such as 1 part per million (ppm) of fluoride in water, which is right around the levels used in US water fluoridation programs, which range from 0.7 to 1.2ppm. Optimizing Gut Flora Strengthens Natural Detoxification Pathways. The presence of, and continual exposure to, toxins is another important factor that can have profound influence on your child IQ both in utero and after birth. Neurological problems such as autism and learning disorders are caused by toxic overload, stemming from abnormal gut flora.

Children use all of their sensory organs to collect information from their environment, which is then passed to the brain for processing. This is a fundamental part of learning. However, in children with Gut and Psychology Syndrome (GAPS), the toxicity flowing from their gut throughout their bodies and into their brains, clogs the brain with toxicity, preventing it from performing its normal function and process sensory information. GAPS may manifest as a conglomerate of symptoms that can fit the diagnosis of autism, attention deficit hyperactivity disorder (ADHD), attention deficit disorder (ADD) without hyperactivity, dyslexia, dyspraxia, or obsessive-compulsive disorder, just to name a few possibilities.

The treatment she developed is called the GAPS Nutritional Program, which covers both diet and detoxification. Fermented foods are a staple of the GAPS diet. The beneficial bacteria in these foods are very potent detoxifiers, capable of drawing a wide range of toxins and heavy metals out of your body. According to Dr. McBride, the GAPS Nutritional Protocol restores the natural detoxification system in about 90 percent of people, and the fermented/cultured foods are instrumental in this self healing process.

The cell wall [of the bacteria] has chelators; molecules that grab hold of mercury, lead, aluminum, and arsenate, they hold them until they are removed through stool. You do not need to consume huge amounts. About a quarter to a half a cup of fermented vegetables, or cultured food such as raw yogurt, per day, is sufficient. The key is variety. The greater the variety of fermented and cultured foods you include in your diet, the better, as each food will inoculate your gut with a variety of different microorganisms.

Phosphatidylserine (PS), a phospholipid that is an essential part of the membranes that surround all your body cells. PS enables cells to move nutrients into and cellular waste out of each living cell in your body. Abundant in your brain and in the membranes of your brain cells, PS is important for brain functions such as memory, judgment, and reasoning. Cow brain, mackerel, herring, and organ meats are some of the foods that contain higher levels of PS 
GPC (Glycerophosphocholine), the bioactive form of choline. Unlike the other forms of choline, GPC is the form that has substantial clinical evidence behind it for its direct effect on healthy brain functions. It occurs naturally in limited quantities in eggs, milk, nuts, fish, certain vegetables, organ meats, and human breast milk Magnesium is another important element needed for optimal brain function and IQ. Stress has been shown to have a detrimental impact on magnesium levels, which in turn can result in lower IQ levels in school-age children, according to some recent published research.
Taking Omega 3, Vitamin DIII, Iodine along with all other essential vitamins, minerals, and amino acids will help you live and thrive to $101++$

\section{Acknowledgements}

None.

\section{Conflict of interest}

The author declares no conflict of interest. 